TITLE:

\title{
Factors promoting maternal trophic egg provisioning in non- eusocial animals
}

AUTHOR(S):

Noriyuki, Suzuki; Kawatsu, Kazutaka; Osawa, Naoya

\section{CITATION:}

Noriyuki, Suzuki ...[et al]. Factors promoting maternal trophic egg provisioning in non-eusocial animals. Population Ecology 2012, 54(3): 455-465

ISSUE DATE:

2012-07

URL:

http://hdl.handle.net/2433/158353

\section{RIGHT:}

The final publication is available at www.springerlink.com; This is not the published version. Please cite only the published version.; この論文 は出版社版でありません。引用の際には出版社版をご確認ご利用くだ さい。 


\section{Title: Factors promoting maternal trophic egg provisioning in}

\section{2 non-eusocial animals}

4 Authors: Suzuki Noriyuki ${ }^{1}$, Kazutaka Kawatsu $^{1}$ and Naoya Osawa ${ }^{2}$

$6 \quad{ }^{1}$ Laboratory of Insect Ecology, Graduate School of Agriculture, Kyoto University

7 Kitashirakawa-oiwake, Sakyo, Kyoto, Japan

8

$9 \quad{ }^{2}$ Laboratory of Forest Ecology, Graduate School of Agriculture, Kyoto University

10 Kitashirakawa-oiwake, Sakyo, Kyoto, Japan

11

$12 *$ Author for correspondence

13 E-mail: nsuzuki@kais.kyoto-u.ac.jp

15 The number of text pages: 27

16 The number of figures: 6

17 The number of tables: 1 


\section{Abstract}

21 The adaptive function of trophic egg-laying is generally regarded as extended parental investment to the offspring. However, the evolutionary factors promoting trophic egg-laying are still unclear, because the amount of maternal investment per offspring

24 should be ideally equal between smaller offspring with trophic eggs and larger offspring without any additional investment. Several authors have suggested that trophic egg-laying should evolve only when egg size is constrained, but this hypothesis has not

27 been evaluated. We investigated the evolutionary mechanisms of trophic egg-laying by two different approaches. First, we evaluated morphological constraints on egg size in two sibling ladybird species, Harmonia axyridis, which is known to produce trophic eggs, and $H$. yedoensis. Second, we theoretically predicted the optimal proportion of trophic

31 eggs to total eggs and egg size in relation to environmental heterogeneity, predictability

32 of environmental quality, and investment efficiency of trophic egg consumption. The

33 intra- and interspecific morphological comparisons suggest that morphological

34 constraints on the evolutionary determination of egg size are weak at best in the two

35 ladybird species. Moreover, we theoretically showed that small egg size and trophic 
37 size plastically. We also showed that even a small reduction in investment efficiency

38 makes a trophic egg strategy unlikely, despite relatively high environmental predictability.

39 We conclude that trophic egg provisioning may be a flexible maternal adaptation to a

40 highly heterogeneous environment rather than a response to a morphological constraint

41 on egg size.

42

43 Keywords Egg size $\cdot$ Environmental heterogeneity $\cdot$ Harmonia $\cdot$ Maternal investment .

44 Morphological constraint · Phenotypic plasticity

45 


\section{Introduction}

Trophic eggs (also called nurse eggs) are non-developing eggs or egg-like structures produced for offspring consumption (Crespi 1992). Strictly speaking, trophic egg-laying

50 is an evolved maternal phenotype, not simply the unavoidable production of

51 non-developing eggs that happen to be eaten by offspring (Crespi 1992, Perry and

52 Roitberg 2006). Trophic egg-laying occurs in diverse animal taxa with various parental

53 care systems (e.g., non-social and eusocial arthropods, gastropods, amphibians, fishes;

54 reviewed by Perry and Roitberg 2006), although it is often confined to only a few species

55 within a taxonomic group (Elgar and Crespi 1992). In general, the adaptive function of

56 trophic egg-laying is regarded as extended parental investment to the offspring

57 (Alexander 1974, Polis 1984). However, the amount of maternal investment per offspring

58 should be ideally equal between smaller offspring with trophic eggs and larger offspring

59 without any additional parental investment (Baur 1990, Dixon 2000). Therefore,

60 evolutionary factors promoting trophic egg-laying, instead of larger offspring size, are

61 still not understood.

62 Several studies have suggested that trophic egg-laying should evolve only when egg

63 size is constrained (Alexander 1974, Polis 1984, Mock and Parker 1997, Dixon 2000). 
64 Morphological constraints on egg size such as the size of the ovipositor or pelvic aperture may prevent small females from producing large eggs (Congdon and Gibbons 1987) and

66 thus may lead to the evolution of trophic egg-laying. Moreover, other hypotheses based

67 on the density effect of competing offspring (Parker and Begon 1986) and the

68 physiological constraints (Sakai and Harada 2001) also explain why larger mothers

69 produce larger offspring. In fact, many field studies of diverse animal taxa have

70 documented positive correlations between maternal size and offspring size (Fox and

71 Czesak 2000). However, no studies have evaluated whether mothers that produce trophic

72 eggs exhibit such constraints on maternal body size.

73 Adaptive mechanisms that might promote trophic egg-laying should be considered

74 in situations where morphological constraints on egg size are not critical. Trophic

75 egg-laying occurs in several groups of eusocial Hymenoptera (Sakagami 1982,

76 Hölldobler and Wilson 1990, Crespi 1992), but it is difficult to examine the adaptive

77 significance of trophic eggs in eusocial systems because complicated conflicts among

78 colony members may obscure the origin and evolution of trophic eggs (Crespi 1992). By

79 contrast, in non-eusocial taxa, the adaptive function of trophic eggs for offspring survival

80 has been examined by focusing on environmental heterogeneity (Kudo and Nakahira

81 2005, Perry and Roitberg 2005a). In fact, studies have documented that females of some 
non-eusocial species that face highly heterogeneous environments adopt a trophic egg

84 between the two strategies, (1) producing small offspring with trophic eggs and (2)

producing large offspring without any additional investment, has not been compared explicitly. For such a comparison, a model that can predict which strategy is evolutionarily stable in a heterogeneous environment needs to be developed. likely to accompany a trophic egg strategy in a heterogeneous environment. As in any and Uller 2007), cues that reliably predict future environment conditions must be present

92 for flexible trophic egg provisioning to evolve. However, the environment that the

93 offspring will face is not always predictable, especially in species in which offspring and

94 adult individuals occupy different niches (Werner and Gilliam 1984, Fischer et al. 2011).

95 Although to reduce the level of uncertainty, mothers can collect information that will be

96 useful in making provisioning decisions (Dall et al. 2005), in a variable environment, a

97 certain level of uncertainty is likely to persist. Moreover, even when mothers can collect

98 information accurately, it may be difficult to compensate for environmental quality in

99 through provisioning without specialized morphology and physiology for trophic egg 
100 production, such as distinct ovariole structure and cellular development. Indeed, in the

101 predatory ladybird Harmonia axyridis which lacks specialized trophic egg structure

102 (Osawa and Yoshinaga 2009), mothers can manipulate the proportion of trophic eggs

103 depending on the prey availability, but the ratio of trophic to viable eggs is variable even

104 in the highly standardized laboratory conditions (Perry and Roitberg 2005a). As a result,

105 the possibility exists that mothers will provision the 'wrong' amount of resources to their

106 offspring. Furthermore, consumption of trophic eggs is likely to involve some waste of

107 maternal resources (Elgar and Crespi 1992). However, no study incorporating such costs

108 and limitations has thus far examined the conditions that favour the trophic egg-laying.

109 In this study, we investigated the evolutionary mechanisms that favour trophic

110 egg-laying by making morphological comparisons and by mathematical modelling. First,

111 we compared egg size and maternal body size both intra- and interspecifically in two

112 sibling ladybird species, Harmonia axyridis Pallas and Harmonia yedoensis Takizawa.

113 Adult body size is quite similar in these two ladybird species (Sasaji 1998), and the

114 hatched larvae of both species eat clusters of sibling eggs, both undeveloped eggs and

115 developing eggs with delayed hatching (Kawai 1978, Osawa 1992a, Osawa and Ohashi

116 2008). The sibling egg consumption by hatchlings can be regarded as an adaptive

117 maternal phenotype in both $H$. axyridis (Perry and Roitberg 2005a) and H. yedoensis 
118 (Noriyuki et al. 2011). If morphological constraints on egg size exist, a positive

119 correlation between egg size and maternal body size would be expected within each

120 species, because smaller mothers cannot produce larger eggs (Fox and Chezak 2000,

121 Fischer et al. 2002, Noriyuki et al. 2010). In addition, if morphological constraints

122 prevent $H$. axyridis females from producing large eggs, then the similar-sized $H$.

123 yedoensis females should not be able to produce eggs larger than those of $H$. axyridis.

124 Furthermore, in females of both species we also examined the ovariole number, which is

125 an important determinant of egg size for a given maternal body size in insects (Gilbert

126 1990, Stewart et al. 1991a). Thus, we evaluated the role of morphological constraints by

127 comparing and assessing morphological traits in these two sibling ladybird species.

128 Second, we constructed a mathematical model to predict the optimal proportion of

129 trophic eggs and egg size that mothers should produce. Under spatially and temporally

130 heterogeneous environments for offspring survival, we investigated how reliable the

131 environmental cues available to the mother have to be for a trophic egg strategy to be

132 favoured by selection. Additionally, we incorporated the fact that a certain proportion of

133 maternal resources provided as trophic eggs are not consumed by the offspring and

134 examined whether natural selection favours trophic egg-laying despite its cost. Finally, on

135 the basis of the results of these different two approaches and the findings of previous 
136 studies, we propose a reasonable explanation for the widespread occurrence of trophic egg-laying in non-eusocial animals.

Methods

140

141 Morphological measurements

143 The generalist predator H. axyridis and the specialist predator H. yedoensis are sibling

144 species with sympatric distributions in central Japan (Noriyuki et al. 2011). Females of

145 the two species produce undeveloped eggs which are consumed by the sibling hatchlings

146 (Osawa and Ohashi 2008). However, the precise developmental mechanisms regulating

147 the production of undeveloped eggs are not fully understood. Indeed, it is possible that

148 gamete incompatibility and sperm limitation cause the failure of fertilization (e.g., Wedell

149 et al. 2002). Moreover, eggs can be infected by male-killing bacteria and killed male

150 embryos appear as infertile eggs in both $H$. axyridis (Majerus et al. 1998) and $H$.

151 yedoensis (N. Osawa, unpublished data), although uninfected females also produce

152 certain proportion of undeveloped eggs. The absence of micropyles is one criterion used

153 to categorize an egg as trophic in the Hymenotera and Heteroptera (e.g., Gobin et al. 1998, 
154 Kudo et al. 2006), but in H. axyridis, micropyles are present in the shells of both developing and undeveloped eggs (Osawa and Yoshinaga 2009). In addition, there is no

156 special feature of the spatial distribution of undeveloped eggs within the clutch in $H$. axyridis (Perry and Roitberg 2005a). However, the proportion of undeveloped eggs increases when $H$. axyridis mothers face the low resource availability (Perry and Roitberg 2005a) and consumption of undeveloped eggs enhance the survival of hatchlings especially when aphid density is low (Osawa 1992a), in a manner consistent with a

161 definition of adaptive trophic egg provisioning (Perry and Roitberg 2006). Although $H$.

162 yedoensis mothers have not been definitely proved to produce trophic eggs in the strict

163 sense (cf. Perry and Roitberg 2006), the consumption of undeveloped eggs greatly

164 enhances prey capture performance in H. yedoensis hatchlings, suggesting that the

165 production of undeveloped eggs has evolved as an adaptive maternal phenotype

166 (Noriyuki et al. 2011). Therefore, even though precise proximate mechanisms have not

167 been identified, the production of undeveloped eggs in $H$. axyridis and $H$. yedoensis can

168 be regarded as an adaptive maternal strategy for the offspring survival.

169 We collected 10 H. axyridis adults at the Botanical Garden of Kyoto University,

170 Kyoto $\left(135^{\circ} 47^{\prime} \mathrm{E}, 35^{\circ} 02^{\prime} \mathrm{N}\right)$, and 25 egg clusters of $H$. yedoensis at Hieidaira, Shiga

$171\left(135^{\circ} 83^{\prime} \mathrm{E}, 35^{\circ} 02^{`} \mathrm{~N}\right)$, in May 2008. We obtained eggs from the adults and the egg 
172 clusters, and reared the resulting offspring at each stage in plastic cups (13 cm wide, 10

$173 \mathrm{~cm}$ high) to the adult stage at $25^{\circ} \mathrm{C}, 16 \mathrm{~L}: 8 \mathrm{D}$, and about $70 \%$ relative humidity. We

174 provided the larvae with a surplus of frozen Ephestia kuehniella Zeller eggs (Beneficial

175 Insectary, Inc., Redding, California, USA). We randomly chose 54 newly-emerged and

176 unmated individuals (27 females and 27 males) of $H$. axyridis and 48 newly-emerged and

177 unmated individuals (24 females and 24 males) of $H$. yedoensis from the stock for the

178 experiment. We used first-generation offspring because (1) there were not enough adults

179 of H. yedoensis in the original field collection for a valid statistical analysis, and (2) it

180 allowed us to use larval morphology to confirm the identities of the two species, which

181 are almost impossible to distinguish on the basis of adult morphology (Sasaji 1998).

182 To obtain eggs from the first-generation adults, we reared mated females

183 individually with a surplus of frozen eggs at $25^{\circ} \mathrm{C}, 16 \mathrm{~L}: 8 \mathrm{D}$, and about $70 \%$ relative

184 humidity. We used 10 eggs from each of five different clutches laid by each female for

185 data analysis, except if the number of eggs in a clutch was less than 10, then all eggs in

186 that clutch were measured. In the case of a female that laid fewer than five clutches, we

187 measured 10 eggs from each clutch that she laid. We measured both egg height $(h)$ and

188 width ( $r$ ) under a stereo microscope (Carl Zeiss ${ }^{\circledR}$ SV-11 APO) to the nearest $0.025 \mathrm{~mm}$.

189 We estimated egg size as the egg volume calculated using the formula $h r^{2} \pi / 6\left(\mathrm{~mm}^{3}\right.$; 
190 Takakura 2004). We measured body length with a slide calipers to the nearest $0.01 \mathrm{~mm}$

191 and used as maternal body size. After a female died or had laid five clutches, we

192 examined her number of ovarioles under the stereo microscope.

193

194 Statistical analysis

195

196 We took into account the fact that the sizes of eggs from the same clutch or laid by the

197 same mothers are not statistically independent. To test for a correlation between maternal

198 body size and egg size, we adopted a regression model with more than one value of the

199 dependent variable (egg size) per value of the independent variable (maternal body size;

200 Sokal \& Rohlf 1995). We used nested analysis of variance (nested ANOVA) with mothers

201 within ladybird species, and with clutches within mothers, to compare egg size between

202 ladybird species. We analyzed the effects of maternal body size and ladybird species on

203 ovariole number by analysis of covariance (ANCOVA). All statistical analyses were

204 carried out with JMP® 7 (SAS Institute Japan).

205

206

The model 
208 We present the simplest theoretical framework for the evolution of trophic egg-laying that

209 incorporates environmental heterogeneity, environmental predictability, and investment

210 efficiency of trophic eggs. We assume that mothers cannot adjust egg size plastically.

211 This assumption is applicable to many kinds of animals because ovariole or pelvic

212 aperture size should remain unchanged in a given individual female. In fact, egg size

213 appears to be inflexible within individual females in many animals, such as land snails

214 (Baur 1988, Baur and Raboud 1988), insects (Stewart et al. 1991a, 1991b, Dixon and Guo

215 1993, Soares et al. 2001), and birds (Christians 2002), and this inflexibility may be tied to

216 ovariole or pelvic aperture size. Even though some species are able to manipulate egg size

217 in response to the quality of the oviposition site (Leather and Burnand 1987, Fox et al.

218 1997, Mizumoto and Nakasuji 2007), plastic adjustment of egg size is uncommon in

219 animal taxa and the degree of the egg size variation seems to be highly constrained (e.g.,

220 Kawecki 1995). In addition to morphological factors, physiological mechanisms of

221 oogenesis may also constrain the immediate adjustment of egg size. Therefore, we

222 assume instead that egg size can evolve to an optimal value and that mothers can produce

223 trophic eggs to deal with an adverse environment. We assume that trophic eggs and viable

224 eggs are the same size, because no general size difference trend has been reported.

225 Moreover, we assume that trophic and viable eggs provide equal food quality for 
226 offspring survival, although possible differences in chemical composition and function

227 between these eggs have not been examined. For simplicity, we assume that the total

228 amount of maternal resources that can be allocated to the offspring as viable eggs and

229 trophic eggs is the same for all females and fixed to 1.

with only two states: good and poor. The relationship between investment per offspring

factors. Moreover, we do not distinguish between spatial and temporal variation. We

234 specify that the minimum viable offspring size is smaller in the good environment than in

235 the poor environment, because an offspring should require fewer resources to become

236 established in the more favourable environment (Fox et al. 1997). We also assume that,

237 for offspring of a given size, offspring fitness is greater in the good environment than in

238 the poor environment (McGinley et al. 1987). Furthermore, we assume that low levels of

239 maternal investment result in zero fitness for offspring because they need a threshold

240 amount of resource to survive, and that fitness approaches an asymptote at high levels of

241 parental investment because offspring cannot make full use of excess resources (Smith

242 and Fretwell 1974, Parker et al. 2002). Thus, we describe the relationship between

243 offspring fitness $S$ and viable egg size in the good environment by $S_{\mathrm{G}}(e)=1-(1 / e)^{2}$ and 
244 in the poor environment by $S_{\mathrm{P}}(e)=1-(k / e)^{2}$, where the subscripts $\mathrm{G}$ and $\mathrm{P}$ indicate the

245 good and the poor environment, respectively, $e$ is viable egg size and trophic egg size, and

$246 k$ is a constant that specifies the difference in quality between the two environments. To

247 make the labels 'good' and 'poor' biologically feasible, we assume $k>1$ such that

248 offspring survive better in a good environment. We assume that individual females

249 experience the two habitat types, good and poor, in the proportion $p$ and $1-p$,

250 respectively.

251 We define environmental predictability, $q$, as the probability that maternal

252 assessment of the environmental quality is correct. Specifically, we assume that when

253 mothers incorrectly assess a poor environment as a good environment, then they do not

254 provision trophic eggs although the offspring may need them to survive. Conversely,

255 when maternal assessment of the good environment is wrong, then mothers may

256 provision unneeded trophic eggs, causing per offspring maternal investment to exceed the

257 optimal value (Table 1). For simplicity, we assume that predictability $q$ is constant across

258 environmental situations. If $q=1$, then mothers can assess the environmental quality

259 perfectly and provision the optimal proportion of trophic eggs in each environment; in

260 contrast, if $q=0.5$, then mothers provision trophic eggs with a probability of 0.5

261 irrespective of the environmental quality. Because $q<0.5$ is not realistic, we consider 
262 only situations with $0.5 \leq q \leq 1$ in the analysis.

264 amount of maternal investment is given by $p(1-q) t+(1-p) q t=t(p+q-2 p q)$, where $t$

265 is the ratio of trophic eggs to the amount of maternal investment when maternal

266 assessment is wrong in the good environment or correct in the poor environment (that is,

267 the amount of resource when mothers 'evaluate' the environment as poor; Table 1).

268 Similarly, the number of viable eggs in an environment that mothers evaluate as poor is

269 given by $\{p(1-q)(1-t)+(1-p) q(1-t)\} / e=\{(1-t)(p+q-2 p q)\} / e$. Hence, per

270 offspring maternal investment in an environment that mothers evaluate as poor is given

271 by $e+t(p+q-2 p q) \delta \div\{(1-t)(p+q-2 p q)\} / e=\{1+\delta t /(1-t)\} e$, where $\delta$ is the

272 investment efficiency of trophic egg consumption, defined as the proportion of the

273 trophic egg amount consumed by the offspring. Because the total amount of maternal

274 investment is fixed to 1 , the amount of maternal investment that is provided as viable eggs

275 in both environments is given by $1-t(p+q-2 p q)$. Therefore, maternal fitness can be

276 described as the product of offspring number times the probability of offspring survival in

277 each environment:

278

$$
W(e, t)=\frac{1-t(p+q-2 p q)}{e}\left\{p\left(q S_{\mathrm{G}}[e]+(1-q) S_{\mathrm{G}}\left[\left(1+\frac{\delta t}{1-t}\right) e\right]\right)+(1-p)\left(q S_{\mathrm{P}}\left[\left(1+\frac{\delta t}{1-t}\right) e\right]+(1-q) S_{\mathrm{P}}[e]\right)\right\}
$$

\section{Results}


281 Morphological comparisons

282

283 Mean female body length was not significantly different between $H$. axyridis (mean \pm SE

$284=7.03 \pm 0.09 \mathrm{~mm}, n=27)$ and $H$. yedoensis $\left(7.24 \pm 0.10 \mathrm{~mm}, n=24\right.$; Student's $t$ test, $t_{49}$

$285=-1.67, P=0.10)$. Female body length and egg volume were not significantly related in

286 either species (linear regression analysis, $H$. axyridis: $F_{1,25}=0.16, P=0.69, r^{2}=0.003 ; H$.

287 yedoensis: $F_{1,22}=0.25, P=0.62, r^{2}=0.005$; Fig. 1 ). Mean egg volume was significantly

288 smaller in H. axyridis (mean $\pm \mathrm{SE}=0.2478 \pm 0.0011 \mathrm{~mm}^{3}, n=1150$ ) than in H. yedoensis

$289\left(0.3481 \pm 0.0013 \mathrm{~mm}^{3}, n=1046\right.$; nested ANOVA, $F_{1,49}=5458.62, P<0.0001$; female

290 code [species]: $d f=49, F=53.9136, P<0.0001$; clutch code [female code] $: d f=187, F=$

$2916.3250, P<0.0001)$. There were significant maternal body size and species effects on

292 ovariole number but no maternal body size $\times$ species interaction was detected (ANCOVA,

293 maternal body size: $F_{1,47}=9.09, P<0.01$; species: $F_{1,47}=96.10, P<0.0001$; interaction:

$294 \quad F_{1,47}=1.63, P=0.21$; Fig. 2 ).

295

296 Model analysis 
298 We present our results as graphical solutions owing to the complexity of the model. We

299 first consider the special case in which the maternal resource in the trophic eggs is

300 completely consumed by the offspring (i.e., $\delta=1$ ). The effects of the proportion of the

301 good environment $(p)$ on the optimal proportion of trophic eggs and on egg size are

302 depicted graphically in Fig. 3. The optimal proportion of trophic eggs relative to $p$ is a

303 convex upward curve (Fig. 3a), and the optimal egg size decreases with $p$ (Fig. 3b).

304 The effects of environmental predictability $(q)$ on the optimal proportion of trophic

305 eggs and on egg size are depicted graphically in Fig. 4. The optimal proportion of trophic

306 eggs is always zero when it is not possible to predict the environment $(q=0.5)$, and it

307 increases as predictability increases (Fig. 4a).

308 The effects of the magnitude of the difference in quality between the good and poor

309 environments $(k)$ on the optimal proportion of trophic eggs and on egg size are depicted in

310 Fig. 5. Both the proportion of trophic eggs and egg size increase with $k$, but the rate of

311 increase in the proportion of trophic eggs decays as $k$ increases (Fig. 5a), whereas optimal

312 egg size increases linearly with $k$ (Fig. 5b).

313 Finally, we consider the case that the trophic egg resource is not completely

314 consumed by the offspring $(0 \leq \delta \leq 1)$. Despite the costs associated with trophic egg

315 consumption, a trophic egg strategy can evolve in heterogeneous environments (Fig. 6a). 
316 In fact, the greater the difference in quality between two environments, the larger the area

317 in the parameter space where a trophic egg strategy is favoured ('trophic egg area', grey

318 and black in Fig. 6). However, trophic egg area severely decreases as investment

319 efficiency decrease, especially when the difference in quality between two environments

320 is small $(k=1.5$, Fig. $6 \mathrm{~b})$.

321

\section{Discussion}

324 Here we present three key findings in trophic egg evolution. First, intra- and interspecific

325 morphological comparisons suggest that maternal body size is at best a weak

326 morphological constraint on egg size in H. axyridis, which has been experimentally

327 proved to produce trophic eggs (Perry and Roitberg 2005a), and H. yedoensis (Fig. 1).

328 Second, we find that trophic egg-laying is expected to evolve in heterogeneous

329 environments when mothers cannot manipulate egg size plastically (Fig. 3a). Third, we

330 theoretically show that a small reduction in investment efficiency in tropic egg

331 consumption greatly reduces the likelihood of trophic egg evolution, even when cue

332 reliability is relatively high (Fig. 6). Taken together, these finding lead us to conclude that

333 maternal adaptation to highly heterogeneous environments rather than morphological 
334 constraints on egg size is a sufficient explanation for the evolution of trophic egg-laying

335

336

337

338

340

341

342

in some non-eusocial animals.

This is the first study to evaluate the role of morphological constraints on egg size in species that produce trophic eggs. We found no significant correlation between egg size and maternal body size in $H$. axyridis or H. yedoensis (Fig. 1), indicating that smaller females can produce eggs similar in size to the eggs of larger females. Moreover, the relationship between egg size and maternal body size was extremely weak in both species, accounting for less than $1 \%$ of the total variation in egg size. Furthermore, $H$. yedoensis females produce larger eggs than $H$. axyridis females, despite the similar maternal body size in the two species (Fig. 1). A reduction in the number of ovarioles should contribute to the production of larger eggs relative to maternal body size (Fig. 2). This result is consistent with the previous finding that species of ladybirds with few ovarioles lay larger eggs than similar-sized species with many ovarioles (Stewart et al. 1991a). These results suggest that maternal body size as a morphological constraint has at best a minor role in the determination of egg size in the two studied ladybird species. Recent studies of insects also suggest that the importance of morphological constraints on the evolution of egg size has been overemphasized (Fischer et al. 2002, Bauerfeind and Fischer 2008, Noriyuki et al. 2010). Importantly, morphological constraints are particularly unlikely to exist when 
352 eggs are small relative to the size of the mother, as is the case in most insect species as

353 well as tree frogs and sharks that produce large numbers of trophic eggs. In addition, even

354 if morphological constraints prevent small females from producing eggs of optimal size,

355 such females can overcome such constraints by producing more elongated eggs

356 (Congdon and Gibbons 1987). In the subsocial bug Adomerus triguttulus, viable eggs are

357 more elongated than trophic eggs (Kudo et al. 2006), suggesting that the females may

358 have potential to overcome morphological constraints on egg size by changing the egg

359 morphology. Moreover, in several reptiles, smaller mothers produce elongated eggs,

360 presumably to facilitate their smooth passage out of the mother's body (Sinervo and Licht

361 1991, Clark et al. 2001, Ji et al. 2006, Rollinson and Brooks 2008). Therefore, it is

362 possible that morphological constraints may not adequately account for trophic egg

363 evolution in other animals.

364 Instead, our theoretical model showed that trophic egg provisioning to small

365 offspring is favoured in heterogeneous environments when mothers cannot manipulate

366 egg size plastically (Fig. 3). Optimal per offspring maternal investment in a poor

367 environment can also be achieved by the evolution of large eggs, without trophic

368 egg-laying, because very large offspring can survive despite variation in environmental

369 quality. As a result, in some circumstances selection favours females that consistently 
370 produce large eggs. Females following this non-plastic strategy, however, are obligated to

371 invest an amount of resources in excess of the optimal value in the good environment,

372 which does not require a large amount of maternal resources. In contrast, by following a

373 trophic egg-laying strategy, females can change their per offspring maternal investment

374 even after deposition of viable eggs, suggesting that trophic egg production and

375 consumption by hatchlings allows females to flexibly adapt to a variable resource

376 environment.

377 Note that the maximum proportion of trophic eggs is predicted when $0.5<p<1$ (Fig.

378 3a). This result indicates that trophic egg-laying tends to be favoured when the proportion

379 of the good environment is higher than the proportion of the poor environment. This

380 result may appear counterintuitive, because we assume that mothers provision trophic

381 eggs to deal with a poor environment. However, although the large egg strategy is

382 inflexible, it can consistently achieve a high offspring survival rate even in a poor

383 environment. By contrast, a trophic egg strategy may lead to large reductions of fitness

384 and offspring survival in a poor environment if the mother incorrectly assesses the

385 environmental quality and therefore fails to provide trophic eggs to small offspring. Thus,

386 a large egg strategy, which should be a safe strategy even in a poor environment, may be

387 favoured when the proportion of poor environment is relatively high. 
Our conclusion that evolution of trophic eggs requires a highly heterogeneous

environment is consistent with the empirical reports in both vertebrates and invertebrates

(e.g., Crump 1981, Dixon 1998). For example, in aphidophagous lacewings and ladybirds,

food resources are frequently and intermittently limited over time because of the

ephemeral nature of aphid colonies (Osawa 1992b, Hemptinne et al. 1992, Dixon 1998),

and they are also spatially heterogeneous in quality and quantity (Osawa 2000). In

394 sub-social animals that provide parental care to offspring even after the hatching (e.g.,

395 tree frogs, burrower bugs, and passalid beetles), as well as predatory animals without

396 effective natural enemies (e.g., sharks and ladybirds), mothers may have relatively long

397 ecological longevity and thus may experience various environmental conditions over

398 their reproductive period. Therefore, it is suggested that trophic egg provisioning may

399 function as a flexible solution for dealing with multiple habitats (Perry and Roitberg

400 2006). Because in our model we do not distinguish between spatial and temporal

401 variation, our findings are potentially applicable to diverse animal taxa producing trophic

402 eggs to cope with predictable environmental variation.

403 Our model revealed that environmental predictability enhances the likelihood that

404 trophic egg provisioning to small eggs will evolve (Fig. 4). Our result indicates that the

405 evolution of trophic egg-laying is possible in heterogeneous environments if mothers 
406 have even a little ability to estimate the offspring's environment ( $q \geq 0.5)$. However, recall

407 that this result holds only when the investment efficiency of trophic egg consumption $\delta$ is

4081 (results with $0 \leq \delta \leq 1$ are discussed below).

409

In some species with trophic egg-laying, mothers are known to evaluate

410 environment conditions that their offspring will face in several ways. For example, in tree

411 frogs, mothers adjust the number of trophic eggs based on the number of offspring (Kam

412 et al. 1998) and offspring age (Gibson and Buley 2004). In A. triguttulus females adjust

413 the number of trophic eggs per viable egg in response to varying resource environments

414 prior to oviposition (Kudo and Nakahira 2005). However, in a majority of trophic egg

415 laying species, the role of environmental cues in trophic egg provisioning has not been

416 examined (Perry and Roitberg 2006). In order to evaluate our model prediction that

417 environmental predictability should be necessary for evolution of trophic egg-laying (Fig.

418 4), empirical tests are required to detect maternal plasticity in trophic egg-laying.

419 Our model also demonstrated that the evolution of trophic eggs is highly sensitive to

420 the wasteful expenditure of maternal resource for trophic eggs even when environmental

421 predictability is relatively high (Fig. 6). In particular, when environmental quality does

422 not differ very much between the good and poor environments ( $k=1.5$ in Fig. 6), even a

423 small reduction of investment efficiency makes the evolution of trophic egg-laying 
424 unlikely. Both nutritive parts of trophic eggs and the shells may be left uneaten by

425 offspring (Perry and Roitberg 2005a). Moreover, non-sibling conspecific individuals and

426 other predators attack trophic eggs, especially in species with no post-natal parental care

427 but also in sub-social animals (Osawa 1989, Nomakuchi et al. 2001). We suggest

428 therefore that these moderate but unavoidable costs associated with trophic egg

429 consumption may mitigate against the evolution of trophic egg-laying, thus accounting

430 for the evolution of trophic egg laying in some taxa and not others.

Variation in the division of maternal resources among siblings may impose both

costs and benefits on maternal trophic egg provisioning, although our model does not

433

explicitly consider this mechanism. Classical optimal investment theory predicts that the

amount of parental resource per offspring should be constant in a given environment

435 (Smith and Fretwell 1974). In real organisms, however, the amount of parental resource

436 per offspring in a brood with trophic eggs frequently varies among siblings (Osawa

437 1992a), because hatching asynchrony and trophic egg location in the clutch, for example,

438 may cause a bias in resource allocation (Osawa 1992a, Perry and Roitberg 2005a).

439 Moreover, adults that abandon their eggs presumably have little ability to control the

440 distribution of resources among offspring. Thus, it is suggested that biases in resource

441 allocation among siblings may prevent mothers from producing trophic eggs. Conversely, 
442 trophic egg-laying may operate as a bet-hedging strategy by generating variation in the

443 size of offspring, the largest of which can survive in the event of unpredictable poor food

444 availability (Perry and Roitberg 2006). In support of this argument, some empirical and

445 comparative studies suggest that within-clutch variation in egg size can reflect an

446 adaptive strategy for dealing with in unpredictable environments in diverse animal taxa

447 such as frogs and fishes (Crump 1981, Einum and Fleming 2004, Marshall et al. 2008,

448 Crean and Marshall 2009). However, the bet-hedging hypothesis for trophic eggs has yet

449 to be tested against the alternative hypothesis of a single optimum in provisioning per

450 offspring. Further investigation of bet-hedging as an evolutionary mechanism promoting

451 trophic egg provisioning should be a productive area of investigation.

452

Our model results are consistent with the findings of previous theoretical studies on adaptive phenotypic plasticity (Berrigan and Scheiner 2004). Specifically, models of

454 phenotypic plasticity generally show that plasticity is favoured when (1) there is

455 environmental heterogeneity (spatial or temporal), (2) there are cues that reliably predict

456 future environmental conditions, and (3) the cost of plasticity is low. Therefore, we

457 suggest that trophic egg provisioning can be regarded as one strategy of adaptive

458 phenotypic plasticity when plastic adjustment of egg size is constrained.

459 In our model, parental-offspring conflict is not taken into account: We assumed that 
460 offspring cannot influence parental behaviour. Importantly, selection typically maximizes

461 maternal rather than offspring fitness, particularly in species with no post-natal parental

462 care, in which offspring counter-strategies may be less likely to evolve (Smith and

463 Fretwell 1974, Trivers 1974). However, parental-offspring conflict affects the evolution

464 of maternal reproductive strategies if offspring can counteract maternal strategies (Parker

465 et al. 2002, Perry and Roitberg 2005b). In particular, Crespi (1992) discussed

466 hypothetically the evolution of trophic eggs in the context of reduction of

467 parent-offspring conflict over sibling cannibalism. Specifically, he suggests that when

468 parent and offspring interests conflict over sibling cannibalism, mothers might adopt a

469 strategy to limit cannibalism by producing trophic eggs, which are less costly than viable

470 eggs but which provide enough energy to cause offspring to refrain from eating viable

471 siblings (Crespi 1992). This hypothesis and our predictions concerning environmental

472 heterogeneity are not necessarily mutually exclusive. Models that incorporate the

473 offspring's point of view need to be developed to predict whether Crespi's (1992)

474 argument can function as a general explanation for trophic egg evolution. However, some

475 empirical data refute the generality of the parent-offspring conflict reduction hypothesis.

476 In particular, Kudo and Nakahira (2004) explicitly rejected the hypothesis by showing in

477 careful experiments in the sub-social burrower bug that the presence or absence of trophic 
478 eggs did not affect the rate of sibling cannibalism. Moreover, a cost difference between

479 trophic and viable egg production may not be common in animal species (Perry and

480 Roitberg 2006). Nevertheless, further studies are needed to identify possible differences

481 in quality between trophic and viable eggs to evaluate the parent-offspring conflict

482 reduction hypothesis. 


\section{Acknowledgement}

484

485 We thank T. Nishida and N. Baba for valuable discussions, Y. Harada for advice on

486 mathematical analyses, S. Seiter for improving English version, and M. Tokeshi and J.-Y.

487 Ide for comments on the manuscript. We are grateful to the staff at the Botanical Garden

488 of Kyoto University for permission to collect ladybirds. This study was supported by a

489 Research Fellowship for Young Scientists from the Japan Society for the Promotion of

490 Science to S. Noriyuki and K. Kawatsu, and a Grant-in-Aid for Scientific Research

491 (Ministry of Education, Culture, Sports, Science and Technology of Japan; No.

492 20405047) to N. Osawa. 


\section{References}

494

495 Alexander RD (1974) The evolution of social behavior. Annu Rev Ecol Syst 5:325-383

496

497 Bauerfeind SS, Fischer K (2008) Maternal body size as a morphological constraint on egg

498 size and fecundity in butterflies. Bas Appl Ecol 9:443-451

499

500 Baur B (1988) Repeated mating and female fecundity in the simultaneously

501 hermaphroditic land snail Arianta arbustorum. Int J Invert Repro Develop 14:197-204

502

503 Baur B (1990) Possible benefits of egg cannibalism in the land snail Arianta arbustorum

504 (L.). Funct Ecol 4:679-684

505

506 Baur B, Raboud C (1988) Life history of the land snail Arianta arbustorum along an

507 altitudinal gradient. J Anim Ecol 57:71-87

508

509 Berrigan D, Scheiner SM (2004) Modeling the evolution of phenotypic plasticity. In:

510 DeWitt TJ, Scheiner SM (eds) Phenotypic plasticity: functional and conceptual 
511 approaches. Oxford University Press, Oxford, pp 82-97

512

513 Christians JK (2002) Avian egg size: variation within species and inflexibility within

514 individuals. Biol Rev 77:1-26

515

516 Clark PJ, Ewert MA, Nelson CE (2001) Physical apertures as constraints on egg size and

517 shape in the common musk turtle, Sternotherus odoratus. Funct Ecol 15:70-77

518

519 Congdon JD, Gibbons JW (1987) Morphological constraint on egg size: a challenge to

520 optimal egg size theory? Proc Natl Acad Sci USA 84:4145-4147

521

522 Crean AJ, Marshall DJ (2009) Coping with environmental uncertainty: dynamic bet

523 hedging as a maternal effect. Phil Trans R Soc B 364:1087-1096

524

525 Crespi BJ (1992) Cannibalism and trophic egg in subsocial and eusocial insects. In: Elgar

526 MA, Crespi BJ (eds) Cannibalism: ecology and evolution among diverse taxa. Oxford 
529 Crump ML (1981) Variation in propagule size as a function of environmental uncertainty

530 for tree frogs. Am Nat 117:724-737

531

532 Dall SRX, Giraldeau LA, Olsson O, McNamara JM, Stephens DW (2005) Information

533 and its use by animals in evolutionary ecology. Trends Ecol Evol 20:187-193

534

535 Dixon AFG (1998) Aphid ecology. Chapman and Hall, London

536

537 Dixon AFG (2000) Insect predator-prey dynamics: ladybird beetles \& biological control.

538 Cambridge University Press, Cambridge

539

540 Dixon AFG, Guo Y (1993) Egg and cluster size in ladybird beetles (Coleoptera:

541 Coccinellidae): the direct and indirect effects of aphid abundance. Eur J Entomol

$54290: 457-463$

543

544 Einum S, Fleming IA (2004) Environmental unpredictability and offspring size: 
547 Elgar MA, Crespi BJ (1992) Cannibalism: ecology and evolution among diverse taxa.

548 Oxford Univesity Press, Oxford

549

550 Fischer K, Zwaan BJ, Brakefield PM (2002) How does egg size relate to body size in

551 butterflies? Oecologia 131:375-379

552

553 Fischer B, Taborsky B, Kokko H (2011) How to balance the offspring quality-quantity

554 tradeoff when environmental cues are unreliable. Oikos 120:258-270

555

556 Fox CW, Czesak ME (2000) Evolutionary ecology of progeny size in arthropods. Annu

557

Rev Entomol 45:341-369

558

559 Fox CW, Thakar MS, Mousseau TA (1997) Egg size plasticity in a seed beetle: an

560 adaptive maternal effect. Am Nat 149:149-163

561

562 Gibson RC, Buley KR (2004) Maternal care and obligatory oophagy in Leptodactylus

563 fallax: a new reproductive mode in frogs. Copeia 2004:128-135 
565 Gilbert F (1990) Size, phylogeny and life-history in the evolution of feeding

566 specialization in insect predators. In: Gilbert F (ed) Insect life cycles: genetics, evolution

567 and co-ordination. Springer, New York, pp 101-124

568

569 Gobin B, Peeters C, Billen J (1998) Production of trophic eggs by virgin workers in the

570 ponerine ant Gnamptogenys menadensis. Physiol Entomol 23:329-336

571

572 Hemptinne JL, Dixon AFG, Coffin J (1992) Attack strategy of ladybird beetles

573 (Coccinellidae): factors shaping their numerical response. Oecologia 90:238-245

574

575 Hölldobler B, Wilson EO (1990) The ants. Harvard University Press, Massachusetts

576

577 Ji X, Du WG, Li H, Lin LH (2006) Experimentally reducing clutch size reveals a fixed

578 upper limit to egg size in snakes, evidence from the king ratsnake, Elaphe carinata.

579 Comp Biochem Physiol A 144:474-478

580

581 Kam YC, Lin CF, Lin YS, Tsal YF (1998) Density effects of oophagous tadpoles of

582 Chirixalus eiffingeri (Anura: Rhacophoridae): importance of maternal brood care. 
583 Herpetologica 54:425-433

584

585 Kawai A (1978) Sibling cannibalism in the first instar larvae of Harmonia axyridis Pallas

586 (Coleoptera: Coccinelidae). Kontyû 46:14-19

587

588 Kawecki TJ (1995) Adaptive plasticity of egg size in response to competition in the

589 cowpea weevil, Callosobruchus maculatus (Coleoptera: Bruchidae). Oecologia

590 $102: 81-85$

591

592 Kudo S, Nakahira T (2004) Effects of trophic-eggs on offspring performance and rivalry

593 in a sub-social bug. Oikos 107:28-35

594

595 Kudo S, Nakahira T (2005) Trophic-egg production in a subsocial bug: adaptive plasticity

596 in response to resource conditions. Oikos 111:459-464

597

598 Kudo S, Nakahira T, Saito Y (2006) Morphology of trophic eggs and ovarian dynamics

599 in the subsocial bug Adomerus triguttulus (Heteroptera: Cydnidae). Can J Zool

$600 \quad 84: 723-728$ 
601

602 Leather SR, Burnand AC (1987) Factors affecting life-history parameters of the pine

603 beauty moth, Panolis flammea (D \& S): the hidden costs of reproduction. Funct Ecol

$604 \quad 1: 331-338$

605

606 Majerus TMO, Majerus MEN, Knowles B, Wheeler J, Bertrand D, Kuznetzov VN, Ueno

607 H, Hurst GDD (1998) Extreme variation in the prevalence of inherited male-killing

608 microorganisms between three populations of Harmonia axyridis (Coleoptera:

609 Coccinellidae). Heredity 81:683-691

610

611 Marshall DJ, Uller T (2007) When is a maternal effect adaptive? Oikos 116:1957-1963

612

613 Marshall DJ, Bonduriansky R, Bussière LF (2008) Offspring size variation within broods

614 as a bet-hedging strategy in unpredictable environments. Ecology 89:2506-2517

615

616 McGinley MA, Temme DH, Geber MA (1987) Parental investment in offspring in

617 variable environments: theoretical and empirical considerations. Am Nat 130:370-398

618 
619 Mizumoto M, Nakasuji F (2007) Egg size manipulation in the migrant skipper Parnara

620 guttata guttata (Lepdoptera: Hesperiidae), in response to different host plants. Popul Ecol

$621 \quad 49: 135-140$

622

623 Mock DW, Parker GA (1997) The evolution of sibling rivalry. Oxford University Press,

624 Oxford

625

626 Nomakuchi S, Filippi L, Hironaka M (2001) Nymphal occurrence pattern and predation

627 risk in the subsocial shield bug, Parastrachia japonensis (Heteroptera: Cydnidae). Appl

628 Entomol Zool 36:209-212

629

630 Noriyuki S, Kishi S, Nishida T (2010) Seasonal variation of egg size and shape in

631 Ypthima multistriata (Lepidoptera: Satyridae) in relation to maternal body size as a

632 morphological constraint. Ann Entomol Soc Am 103:580-584

633

634 Noriyuki S, Osawa N, Nishida T (2011) Prey capture performance in hatchlings of two

635 sibling Harmonia ladybird species in relation to maternal investment through sibling

636 cannibalism. Ecol Entomol 36:282-289 
638 Osawa N (1989) Sibling and non-sibling cannibalism by larvae of a lady beetle Harmonia

639 axyridis Pallas (Coleoptera: Coccinellidae) in the field. Res Popul Ecol 31:153-160

640

641 Osawa N (1992a) Sibling cannibalism in the ladybird beetle Harmonia axyridis Pallas:

642 fitness consequences for mothers and offspring. Res Popul Ecol 34:45-55

643

644 Osawa N (1992b) A life table of the ladybird beetle Harmonia axyridis Pallas (Coleoptera,

645 Coccinellidae) in relation to the aphid abundance. Jap J Entomol 60:575-579

646

647 Osawa N (2000) Population field studies on the aphidophagous ladybird beetle Harmonia

648 axyridis (Coleoptera: Coccinellidae): resource tracking and population characteristics.

649 Popul Ecol 42:115-127

650

651 Osawa N, Ohashi K (2008) Sympatric coexistence of sibling species Harmonia yedoensis

652 and H. axyridis (Coleoptera: Coccinellidae) and the roles of maternal investment through

653

egg and sibling cannibalism. Eur J Entomol 105:445-454

654 
655 Osawa N, Yoshinaga A (2009) The presence of micropyles in the shells of developing and

656 undeveloped eggs of the ladybird beetle Harmonia axyridis (Coleoptera: Coccinellidae).

657 Eur J Entomol 106:607-610

658

659 Parker GA, Begon M (1986) Optimal egg size and clutch size: effects of environment and

660 maternal phenotype. Am Nat 128:573-592

661

662 Parker GA, Royle NJ, Hartley IR (2002) Intrafamilial conflict and parental investment: a

663 synthesis. Philos Trans R Soc B 357:295-307

664

665 Perry JC, Roitberg BD (2005a) Ladybird mothers mitigate offspring starvation risk by

666 laying trophic eggs. Behav Ecol Sociobiol 58:578-586

667

668 Perry JC, Roitberg BD (2005b) Games among cannibals: competition to cannibalize and

669 parent-offspring conflict lead to increased sibling cannibalism. J Evol Biol 18:1523-1533

670

671 Perry JC, Roitberg BD (2006) Trophic egg laying: hypotheses and tests. Oikos

$672 \quad 112: 706-714$ 
674 Polis GA (1984) Intraspecific predation and "infant killing" among invertebrates. In:

675 Hausfater G, Hrdy SB (eds) Infanticide: comparative and evolutionary perspectives.

676 Aldine De Gruyter, Berlin, pp 87-104

677

678 Rollinson N, Brooks R (2008) Optimal offspring provisioning when egg size is

679 "constrained": a case study with the painted turtle Chrysemys picta. Oikos 117:144-151

680

681 Sakagami SF (1982) Stingless bees. In: Hermann HR (ed) Social insects, vol. 3.

682 Academic Press, Massachusetts, pp 362-424

683

684 Sakai S, Harada Y (2001) Why do large mothers produce large offspring? Theory and a

685

test. Am Nat 157:348-359

686

687 Sasaji H (1998) Natural history of the ladybirds. University of Tokyo Press, Tokyo (in 688 Japanese)

689

690 Sinervo B, Licht P (1991) Hormonal and physiological control of clutch size, egg size, 
691 and egg shape in side-blotched lizards (Uta stansburiana): constraints on the evolution of

692 lizard life histories. J Exp Zool 257:252-264

693

694 Smith CC, Fretwell SD (1974) The optimal balance between size and number of offspring.

695 Am Nat 108:499-506

696

697 Soares AO, Coderre D, Schanderl H (2001) Fitness of two phenotypes of Harmonia

698 axyridis (Coleoptera: Coccinellidae). Eur J Entomol 98:287-293

699

700 Sokal RR, Rohlf FJ (1995) Biometry, 3rd edition. Freeman, New York

701

702 Stewart LA, Hemptinne JL, Dixon AFG (1991a) Reproductive tactics of ladybird beetles:

703 relationship between egg size, ovariole number and developmental time. Funct Ecol

$704 \quad 5: 380-385$

705

706 Stewart LA, Dixon AFG, Ruzicka Z, Iperti G (1991b) Clutch and egg size in ladybird

707 beetles. Entomophaga 36:93-97

708 
709 Takakura K (2004) Variation in egg size within and among generations of the bean weevil,

710 Bruchidius dorsalis (Coleoptera, Bruchidae): effects of host plant quality and paternal

711 nutritional investment. Ann Entomol Soc Am 97:346-352

712

713 Trivers RL (1974) Parent-offspring conflict. Am Zool 14:249-264

714

715 Wedell N, Gage MJG, Parker GA (2002) Sperm competition, male prudence and

716 sperm-limited females. Trends Ecol Evol 17:313-320

717

718 Werner EE, Gilliam JF (1984) The ontogenetic niche and species interactions in size structures populations. Annu Rev Ecol Syst 15:393-425 


\section{$720 \quad$ Figure legends}

721

722 Fig. 1. Relationship between female body size and egg size (mean \pm SE) by species.

723 Mean volumes of egg produced by $H$. axyridis (open circles) and $H$. yedoensis (closed

724 circles) females of a given body length.

725

726 Fig. 2. Relationship between female body size and number of ovarioles in H. axyridis

727 (open circles) and H. yedoensis (closed circles). Dotted and solid lines represent the linear

728 regression for $H$. axyridis and $H$. yedoensis, respectively.

729

730 Fig. 3. Optimal proportion of trophic eggs (a) and optimal offspring size (b) as a function

731 of the proportion of the good environment. Parameter values used are $k=2.0, q=0.75$

732 (solid line); $k=4.0, q=0.75$ (dashed line); and $k=2.0, q=0.90$ (dotted line); where $k$ is

733 the degree of difference between the two environments and $q$ is environmental

734 predictability.

735

736 Fig. 4. Optimal proportion of trophic eggs (a) and optimal offspring size (b) as a function

737 of environmental predictability. We did not evaluate the situation where $q<0.5$ because it 
738 is biologically unrealistic. Parameter values used are $k=2.0, p=0.50$ (solid line); $k=4.0$,

$739 p=0.50$ (dashed line); and $k=2.0, p=0.25$ (dotted line); where $k$ is the difference

740 between the two environments and $p$ is the proportion of the good environment.

741

742 Fig. 5. Optimal proportion of trophic eggs (a) and optimal offspring size (b) as a function

743 of the difference in quality between the good and bad environments. Parameter values

744 used are $p=0.5, q=0.66$ (solid line); $p=0.25, q=0.66$ (dashed line); and $p=0.5, q=$

7450.75 (dotted line); where $p$ is the proportion of the good environment and $q$ is

746 environmental predictability.

747

748 Fig. 6. Conditions that favour a trophic egg strategy $(t>0)$ when $k=1.5$ (black area) or $k$

$749=2.0$ (black and grey areas), depending on the investment efficiency $\delta$ (horizontal axis):

750 (a) proportion of good environment $p$ (vertical axis), and (b) environmental predictability

$751 q$ (vertical axis). Other parameters: (a) $q=0.75$, (b) $p=0.25$. In the shaded parameter area,

752 a trophic egg-laying strategy is expected to never evolve. 


\section{Table}

Table 1. Relationship between environmental conditions and maternal investment

\begin{tabular}{llll}
\hline Environmental quality & Environmental predictability & Maternal strategy & Per offspring maternal investment* \\
\hline Good $(p)$ & Correct $(q)$ & Viable eggs only & $e$ \\
& Wrong $(1-q)$ & Trophic egg provisioning (if necessary) & $\{1+\delta t /(1-t)\} e$ \\
\multirow{2}{*}{ Poor $(1-p)$} & Correct $(q)$ & Trophic egg provisioning (if necessary) & $\{1+\delta t /(1-t)\} e$ \\
& Wrong $(1-q)$ & Viable eggs only & $e$ \\
\hline
\end{tabular}

*Model parameters $e, t$, and $\delta$ describe the size of viable eggs, the proportion of trophic eggs, and the investment efficiency of trophic eggs, respectively (see text for details). 


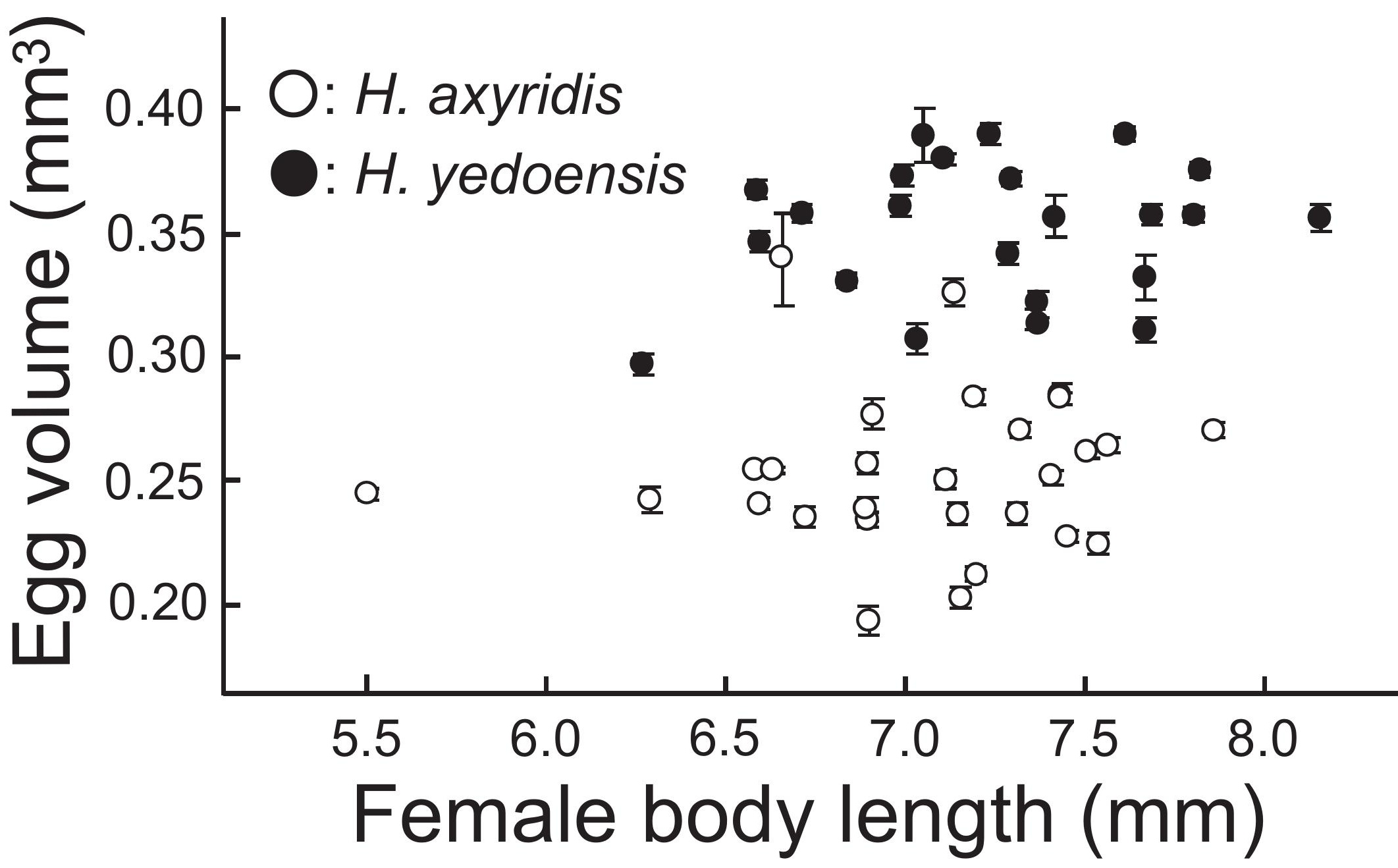




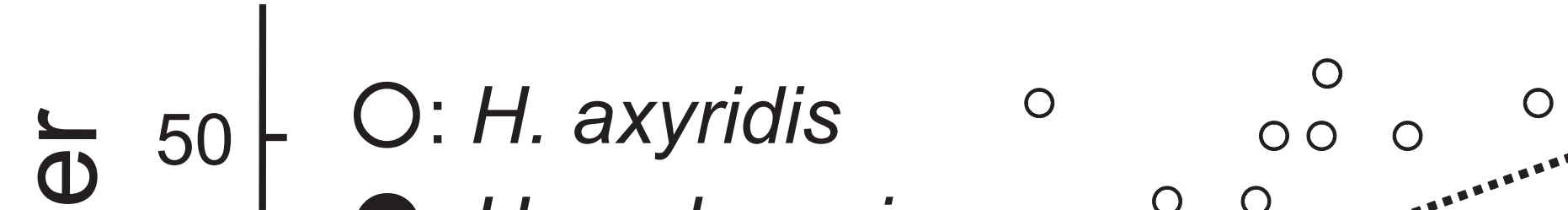
孚
$\underline{\xi}$
$\frac{0}{0}$ O: H. yedoensis<smiles>CCOCCOCCOCCO</smiles><smiles>[TeH4]</smiles>
35
30
25
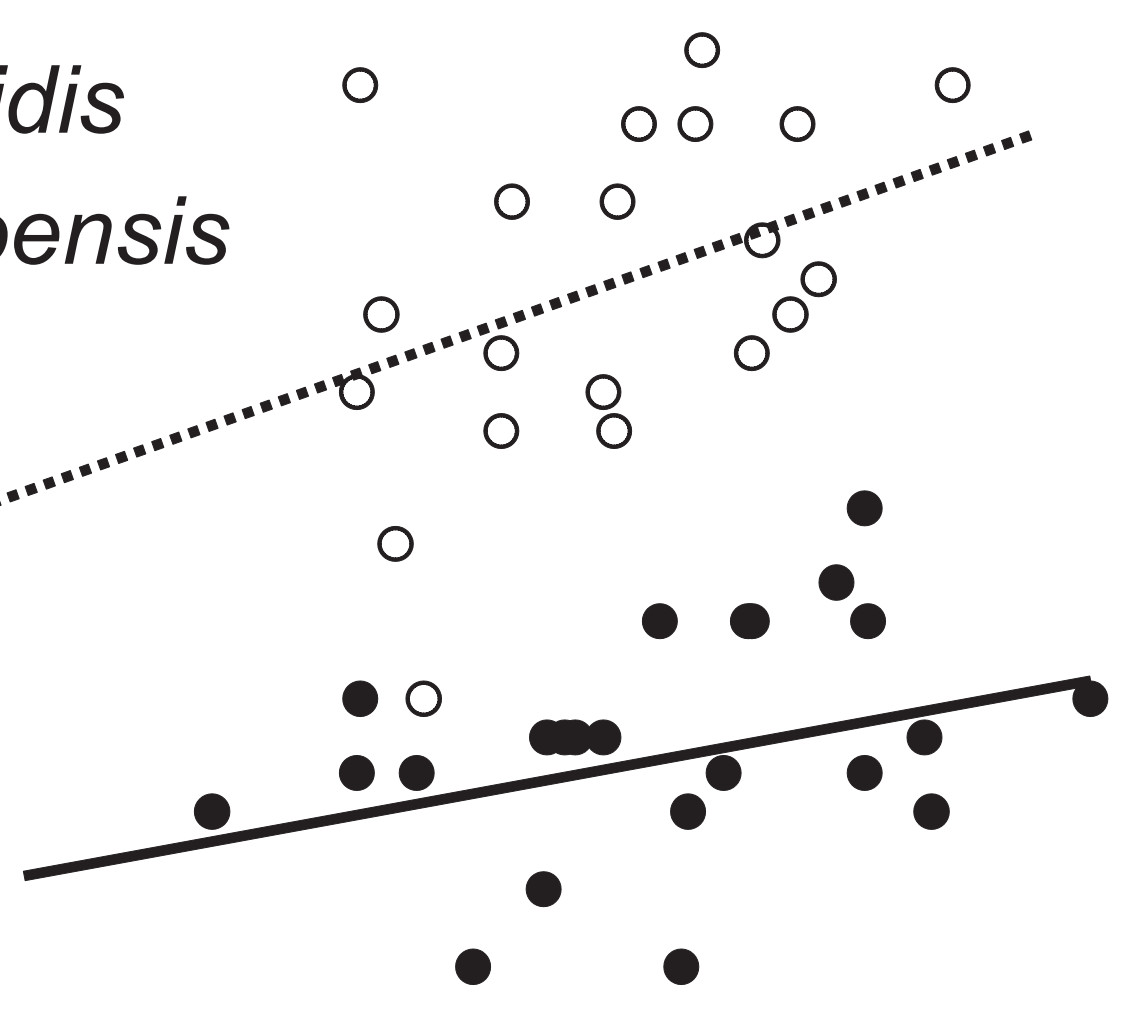

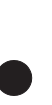

\section{$\begin{array}{lllllll}5.0 & 5.5 & 6.0 & 6.5 & 7.0 & 7.5 & 8.0\end{array}$ Female body length (mm)}

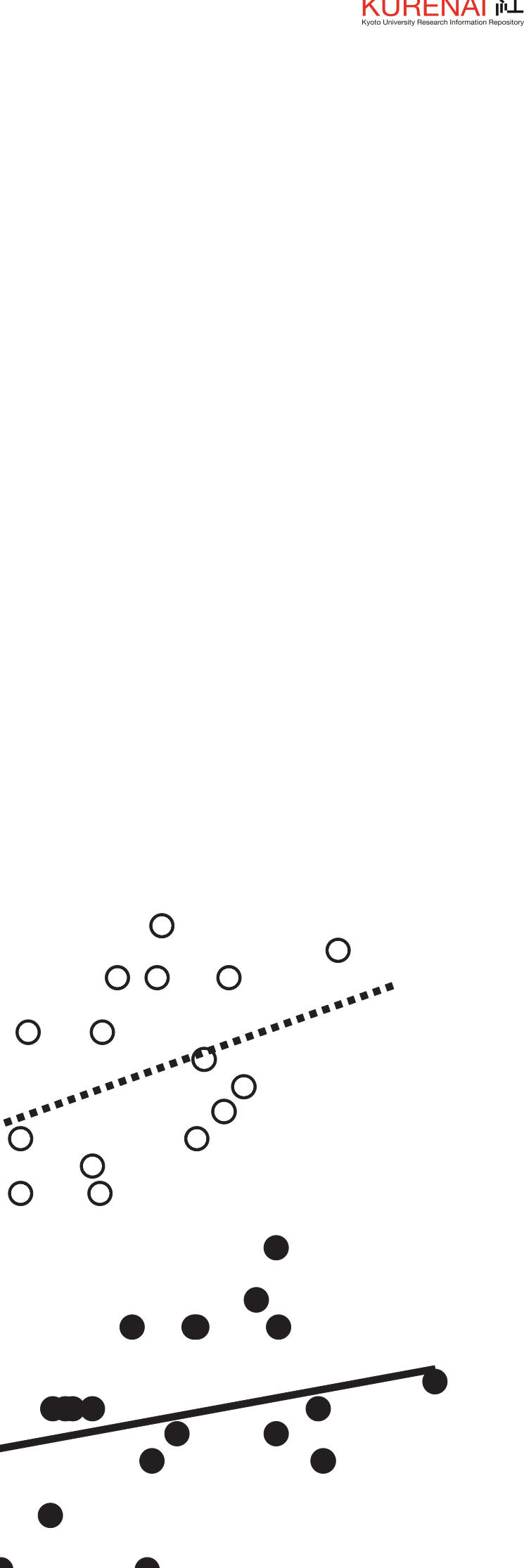



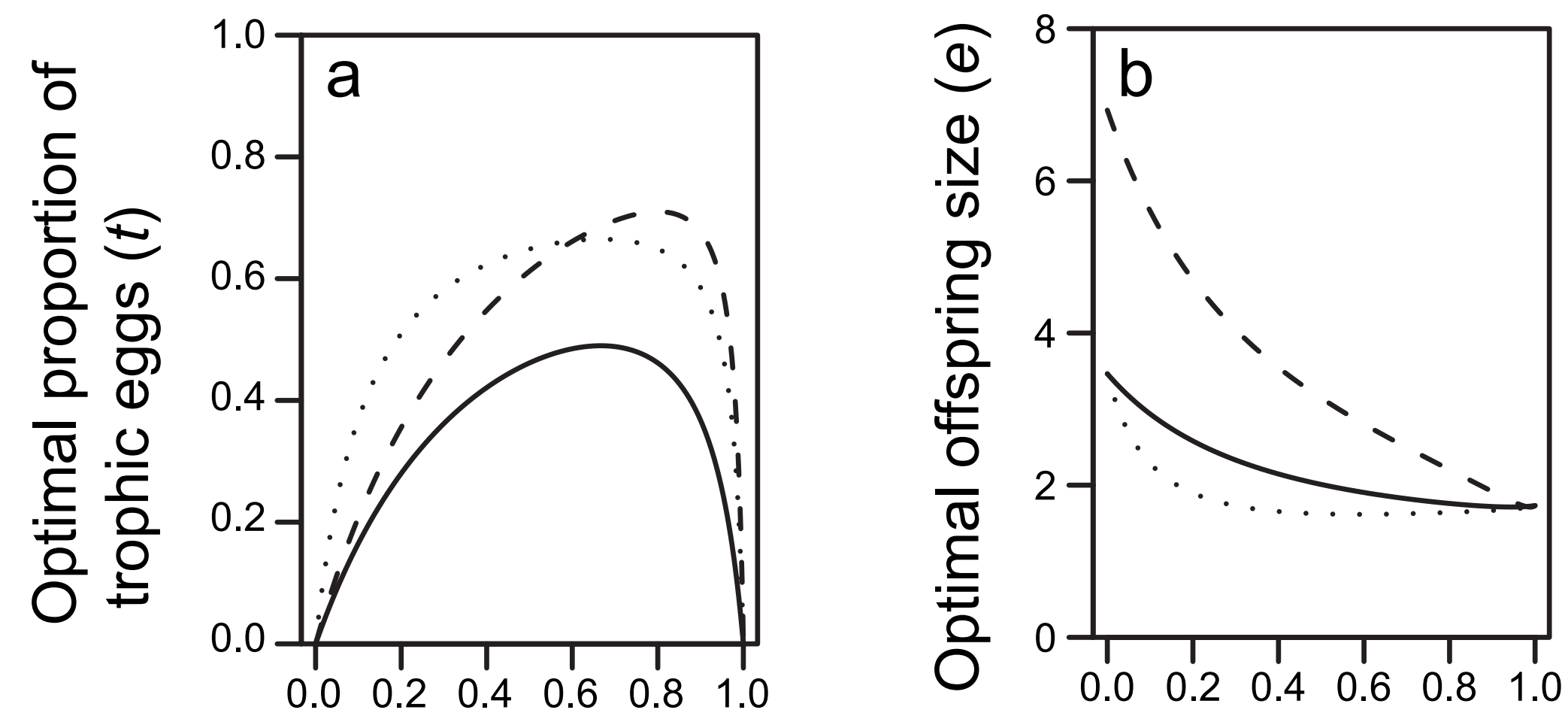

\section{Proportion of the good environment $(p)$}



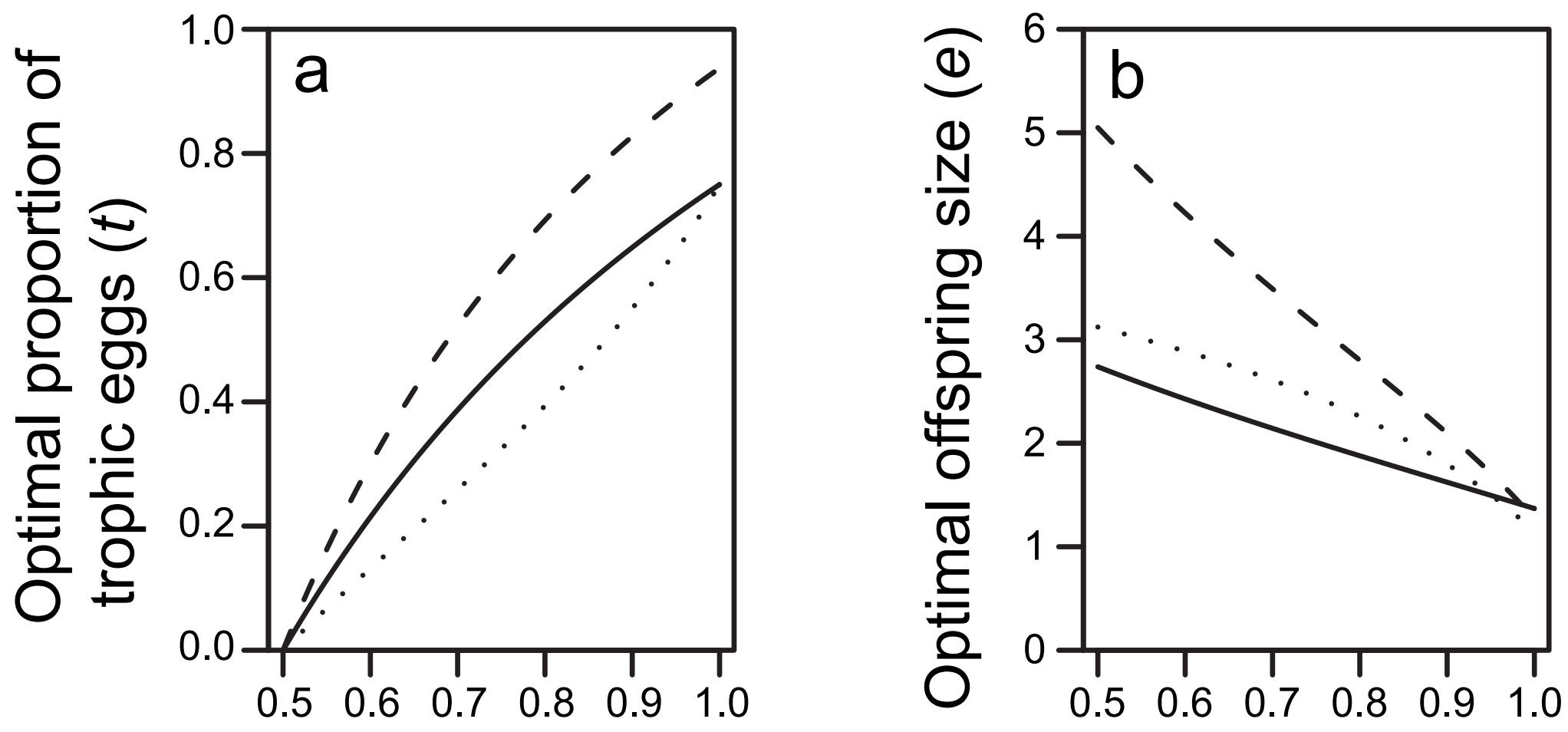

Environmental predicability $(q)$ 

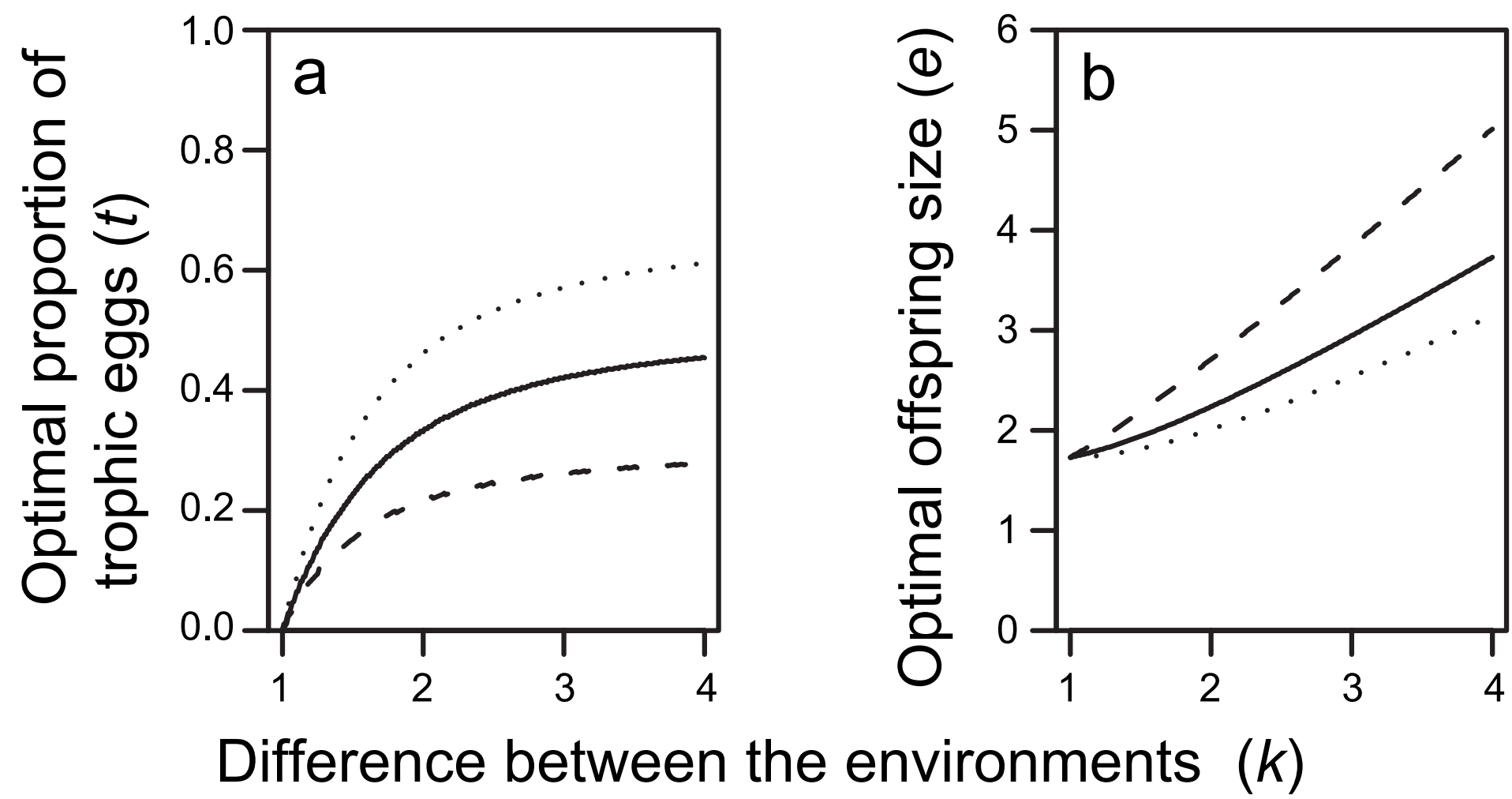

Difference between the environments $(k)$ 


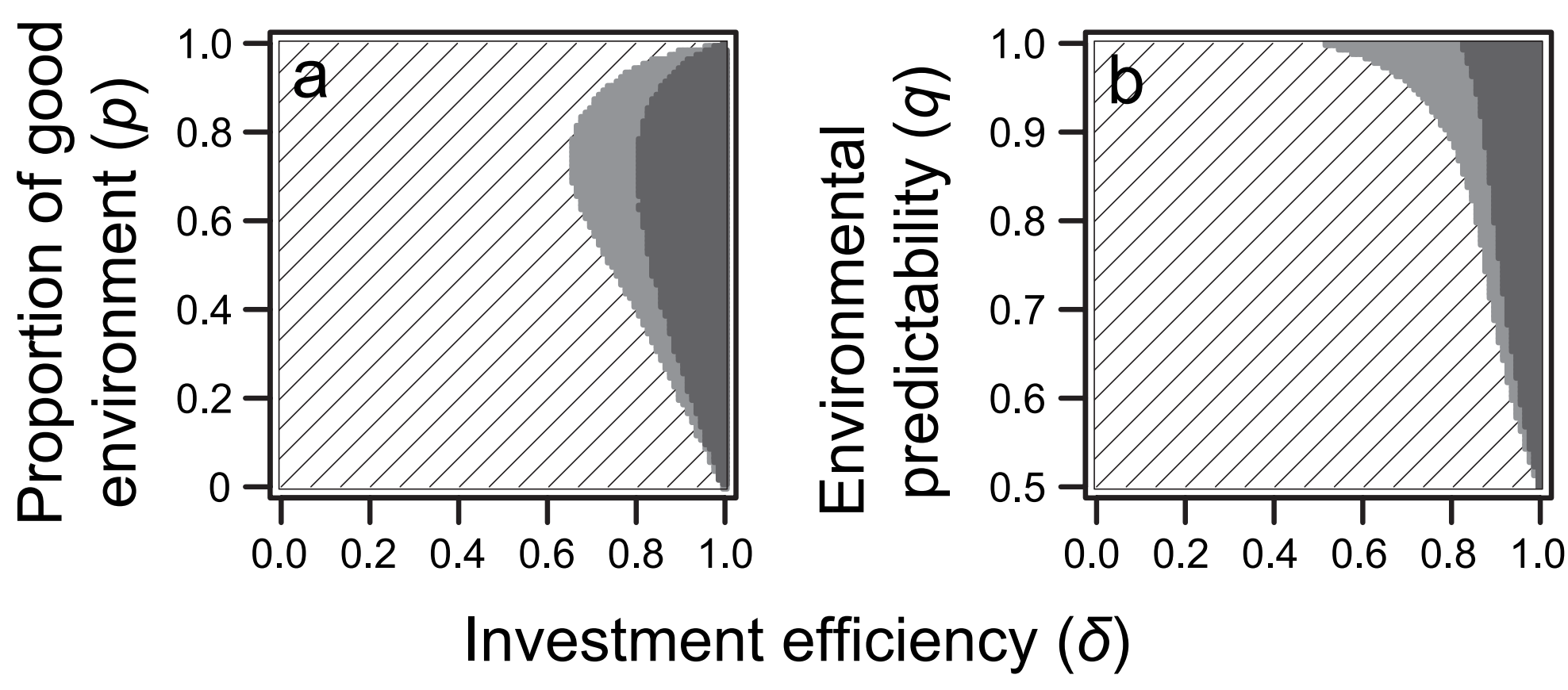

